\title{
Synthesis of Optical Quality ZnO Nanowires Utilizing Ultrasonic Spray Pyrolysis
}

Myo Than Htay, Yuji Tani, Yoshio Hashimoto, and Kentaro Ito

Department of Electrical and Electronic Engineering, Faculty of Engineering, Shinshu University, 4-17-1 Wakasato, Nagano 380-8553, Japan

Keywords: $\mathrm{ZnO}$, Nanowires, Ultrasonic spray pyrolysis

Abstract $\mathrm{ZnO}$ nanowires were grown by an ultrasonic spray pyrolysis technique. The aspect ratio and size of the wire were dependent mainly on the $\mathrm{pH}$ value of a precursor solution and the growth temperature. By high resolution transmission electron microscopic analysis and photoluminescence measurements, it was confirmed that the nanowires are monocrystalline with good optical quality. 


\section{Introduction}

A II-VI compound semiconductor, $\mathrm{ZnO}$, is an environment-friendly material with a direct band gap of $3.37 \mathrm{eV}$ at $300 \mathrm{~K}$ [1]. It has an exciton binding energy of $60 \mathrm{meV}$, which is considerably large compared with those of the other II-VI compounds such as CdS, CdSe, ZnSe, and ZnTe. Because of the large binding energy, the exciton states are stable even at room temperature. Another interesting characteristic of $\mathrm{ZnO}$ is that it can be grown in various crystalline forms with submicron size such as whiskers, nanobelts, nanorods, nanowires, nanoplatelets, and so on. According to these features, $\mathrm{ZnO}$ is a promising candidate for exciton-related optoelectronic devices in the ultraviolet region, and it is also applicable to the devices based on reduced dimensional quantum effect. The stimulated emission and optically-pumped laser action have indeed been demonstrated in $\mathrm{ZnO}$ epitaxial films [2-4], as well as in nanowires [5], at room temperature. However, in order to realize commercially available $\mathrm{ZnO}$-based light emitting devices, development of low-cost and mass-producing techniques for high optical quality $\mathrm{ZnO}$ crystals is still required.

Recently, we succeeded in preparing the $\mathrm{ZnO}$ submicron crystals such as nanowires, nanorods and nanoplatelets in a reproducible manner at a relatively low temperature by a home-made ultrasonic spray pyrolysis (USP) system [6]. This USP technique is an important fabrication technique because it has less complexity, and it is capable to realize low-cost and mass-producing system. In this paper, we discuss the structural properties and optical quality of $\mathrm{ZnO}$ nanowires prepared by this economically viable USP technique.

\section{Experimental details}

In order to prepare $\mathrm{ZnO}$ nanowires, we used zinc acetate as a precursor salt for the zinc source, and de-ionized water as a solvent and an oxygen source. Low cost soda-lime glass was used as a substrate for the growth of nanowires. We added indium nitrate as a metal catalyst in precursor solution which improved the unidirectional growth of the wurtzite crystal. To stabilize the $\mathrm{pH}$ of precursor solution we used ammonium acetate, or a mixture of sodium hydroxide and ethanoic acid as a buffer agent. The details of our USP system were described elsewhere [6].

Crystal surface morphology was studied using a field emission scanning electron microscope (FE-SEM, Hitachi, S-4100). For detailed structural analysis, transmission electron microscopy (TEM, JEOL, JEM-2010, $\mathrm{LaB}_{6}$ cathode) and electron diffraction analysis were carried out at an acceleration voltage of $200 \mathrm{kV}$.

Optical quality of nanowires was evaluated by photoluminescence (PL) measurements. A fourth harmonics (266 nm) of a $Q$-switched Nd:YAG laser with a spot size of $1 \mathrm{~mm}^{2}$ was used as an excitation light source. In detection system, a $0.32 \mathrm{~m}$ monochromator and a gated intensified charge-coupled device (ICCD) camera (Hamamatsu, C5909-06) were used. Spectral resolution of the detection system is $0.5 \mathrm{~nm}$. A setup for the PL measurements was fully described in reference 7 .

\section{Results and discussion}

In our USP technique, the growth temperature $\left(T_{\mathrm{g}}\right)$ and $\mathrm{pH}$ value of a precursor solution are critical parameters in controlling the growth size and aspect ratio (ratio of height to width) of 
nanowires. In Fig. 1(a) and 1(b), FE-SEM images of the wires grown at different temperatures of 300 and $400{ }^{\circ} \mathrm{C}$ for 120 min from the precursor solution with $\mathrm{pH}$ value of 7.2 are shown. The stabilization of $\mathrm{pH}$ value at 7.2 is obtained by adding ammonium acetate $(1.5 \mathrm{M})$ to the precursor solution containing zinc acetate $(0.3 \mathrm{M})$. The average thickness of the wires prepared at $300{ }^{\circ} \mathrm{C}$ is about $100 \mathrm{~nm}$ and their thickness uniformity is fairly good. The aspect ratio is about 100 . At $T_{\mathrm{g}}$ of $400{ }^{\circ} \mathrm{C}$, however, the average thickness increases to a value several times as much, the thickness uniformity worsens, and the aspect ratio is smaller than 40. By comparison, it is recognized that the growth rate perpendicular to the wire axis is faster at a higher $T_{\mathrm{g}}$ so that much bulky crystal wires are obtained at $400{ }^{\circ} \mathrm{C}$. The FE-SEM images of wires grown for $120 \mathrm{~min}$ from the precursor solution with $\mathrm{pH}$ value of 6.2 at different temperatures of 300 and $400{ }^{\circ} \mathrm{C}$ are shown in Fig. 1(c) and 1(d), respectively. To obtain the $\mathrm{pH}$ value of 6.2 , a mixture of sodium hydroxide and ethanoic acid $(0.025$ $\mathrm{M}: 1 \mathrm{~mL}$ ) is dissolved into the precursor solution. For the wires grown at $300{ }^{\circ} \mathrm{C}$, it is found that the average thickness is about $200 \mathrm{~nm}$ with moderate thickness uniformity. The aspect ratio is not larger than 10, which is quite small compared to those grown at the $\mathrm{pH}$ value of 7.2 as discussed above. Again, at a higher $T_{\mathrm{g}}$ of $400{ }^{\circ} \mathrm{C}$, thicker wires are obtained as shown in Fig. 1(d). The average thickness is about 3 times larger than those grown at $300{ }^{\circ} \mathrm{C}$. However, the thickness uniformity and the aspect ratio are lower.

By comparing the crystal topologies of wires prepared under different $\mathrm{pH}$ values, we observed that the aspect ratio of wires synthesized from a more acidic precursor solution is much smaller in average. In other words, unidirectional crystal growth is much preferred in a weaker acidic precursor solution. It is also found that the growth density of wires is higher in those prepared from a solution with low acidity, i.e., the growth rate is enhanced as the solution is approached to neutral. However, when an alkaline precursor solution is used, atomizing process of the solution is not efficient and the growth of crystal wires is not successful because precipitation of colloidal species takes place in the solution during nebulization process. In the case of strong acidic solution, formation of wires is not observed. In our growth technique, the growth temperature necessary for synthesizing nanowires is about $300^{\circ} \mathrm{C}$, which is much lower than those needed in the other growth techniques based on vapour-liquid-solid mechanism using $\mathrm{Au}$ as a catalyst [8]. A possible growth mechanism of our wire structures is proposed elsewhere [6].

Based on structural investigation of these wires, we can classify them into three different types. In Fig. 2(d), 2(h), and 2(l), we depicted the three possible atomic topologies that construct the three distinct types of $\mathrm{ZnO}$ wires. These wires really grow along one of the [0001], [1010], and [1120] crystallographic directions of the wurtzite crystal. We denote these three types of wires as type A, type $\mathrm{B}$, and type $\mathrm{C}$ wire, respectively. Based on TEM and selected area electron diffraction (SAED) analysis, we confirmed that the individual wire is a monocrystalline wurtzite crystal grown in one of the three different crystallographic directions as described above. In Fig. 2(b), 2(f), and 2(j), bright field TEM images of type A, B, and C wires are shown. Here, the tip of type A wire is flat and its cross section is hexagonal in shape. However, the tips of type $\mathrm{B}$ and $\mathrm{C}$ wires consist of three and two facets, respectively and both of them have a rectangular cross section. The SAED patterns obtained from the individual wire with different structural appearances are shown in Fig. 2(a), 2(e), and 2(i). The SAED pattern obtained from [ $\overline{1} 2 \overline{1} 0]$ zone axis of type A wire exhibits periodically arranged 
diffraction spots with two-fold symmetry. Such a kind of diffraction pattern is consistent with the diffraction caused by the planes that are perpendicular to the (0001) plane of wurtzite crystal. On the other hand, the SAED patterns obtained from [0001] zone axis of the type B and C wires show diffraction spots with six-fold symmetry. These results are, of course, consistent with the diffraction originated from the hexagonal close-packed arrangement of lattice points. The high resolution TEM (HRTEM) photographs shown in Fig. 2(c), 2(g), and 2(k) represent the lattice images of nanowires clearly. They coincide quite well with the predicted atomic topologies for each crystal plane as shown in Figs 2(d), (h) and (1). The result indicates how the external structural appearance of nanowires is related to the atomic topology. The lattice spacing are $0.536,0.287$ and $0.169 \mathrm{~nm}$ for (0001), $\{10 \overline{1} 0\}$, and $\{11 \overline{2} 0\}$ planes, respectively.

The PL spectra taken at 8 and $300 \mathrm{~K}$ for thin and thick wires are shown in Fig. 3. Here nanowires with thickness less than $200 \mathrm{~nm}$ are represented by "thin wires", while larger ones by "thick wires". The FWHM of the sharp emission band $(3.36 \mathrm{eV})$ for thin wires at $8 \mathrm{~K}(300 \mathrm{~K})$ is about 20 (140) $\mathrm{meV}$ which is comparable to those prepared by molecular beam epitaxial techniques $[4,9,10]$. We attributed this band to the emission originated from the bound exciton complexes (BEC). There is no deep level emission around $2.45 \mathrm{eV}$ corresponding to oxygen vacancies, but only the excitonic emission at the near band edge region in all the spectra. Recently we confirmed inelastic exciton-exciton scattering emission, which leads to a quadratic excitation-density dependence of emission intensity in our $\mathrm{ZnO}$ nanowires with an average thickness of about $100 \mathrm{~nm}$ $[11,12]$. The result is re-described in Fig. 4. The integrated PL intensities of BEC and P-band are plotted by open and filled circles, respectively as shown in the inset. From the excitation-density dependent PL spectra, it is found that an intense PL emission (denoted as P) at the lower energy side of BEC band appears as the excitation density is increased. This P-band emission is considered to be originated from inelastic exciton-exciton scattering process. Since, it exhibits red-shifting and enhances quadratically with the excitation density, while the BEC emission obeys a linear dependency without energy shifting. The observation of such exciton-exciton scattering process has not been reported for $\mathrm{ZnO}$ nanowires grown by USP technique, although there are some articles on such nonlinear optical process in high quality films prepared by other methods $[4,9,10]$. Hence, it is recognized that due to good crystal quality, the exciton-exciton scattering process is efficient in thin wires. However, in thick wires, the exciton-related emission is weaker, indicating that the optical quality of the thin wires is much better than the thick ones.

\section{Conclusions}

High optical quality $\mathrm{ZnO}$ nanowires, which exhibited sharp and narrow excitonic PL emission at $3.36 \mathrm{eV}$, were synthesized by an economically viable USP method. The nanowires with an average thickness of $100 \mathrm{~nm}$ exhibits strong PL emission at about $3.32 \mathrm{eV}$ due to inelastic exciton-exciton scattering process under moderate density of excitation. Clear lattice images for the nanowires were obtained by high resolution transmission electron microscopy. They revealed directly that monocrystalline nanowires grow parallel or perpendicular to the [0001] axis depending on the deposition condition. For wire growth, the proper $\mathrm{pH}$ value of precursor solution was in the range of 6.2 and 7.2. The aspect ratio of wires increased as the $\mathrm{pH}$ value of precursor solution approached the 
neutral. A fairly low growth temperature between 300 and $400{ }^{\circ} \mathrm{C}$ was suitable for the fabrication of wires.

\section{Acknowledgements}

One of the authors (M. T. Htay) would like to express gratitude to the Ministry of Education, Culture, Sports, Science and Technology of Japan for grant of postgraduate scholarship. Special thanks are also due to Mr. Tomohiko Yamakami for TEM photography, and Mr. Isamu Minemura for his neat work on the construction of experimental apparatus. Especially, we would like to thank Prof. Minoru Itoh for his kind advice, and Mr. Daisuke Iri and Mr. Tsuyoshi Katagiri for their great help in the PL measurements. 


\section{References}

1. N. Izyumskaya, V. Avrutin, Ü. Özgür, Y. I. Alivov, H. Morkoç, Phys. Stat. Sol. (b) 244, 1439 (2007)

2. D. M. Bagnall, Y. F. Chen, Z. Zhu, T. Yao, M. Y. Shen, T. Goto, Appl. Phys. Lett. 73, 1038 (1998)

3. D. M. Bagnall, Y. F. Chen, Z. Zhu, T. Yao, S. Koyama, M. Y. Shen, T. Goto, Appl. Phys. Lett. 70, 2230 (1997)

4. P. Zu, Z. K. Tang, G. K. L. Wong, M. Kawasaki, A. Ohtomo, H. Koinuma, Y. Segawa, Solid State Commun. 103, 459 (1997)

5. M. H. Haung, S. Mao, H. Feick, H. Yan, Y. Wu, H. Kind, E. Weber, R. Russo, P. Yang, Science 292, 1897 (2001)

6. M. T. Htay, Y. Hashimoto, K. Ito, Jpn. J. Appl. Phys. 46, 440 (2007)

7. M. Itoh, T. Sakurai, Phys. Rev. B 73, 235106 (2006)

8. M. H. Huang, Y. Wu, H. Feick, N. Tran, E. Weber, P. Yang, Adv. Mater. 13, 113 (2001)

9. A. Yamamoto, K. Miyajima, T. Goto, H. J. Ko, T. Yao, J. Appl. Phys. 90, 4973 (2001)

10. H. J. Ko, Y. F. Chen, Z. Zhu, T. Yao, I. Kobayashi, H. Uchiki, Appl. Phys. Lett. 76, 1905 (2000)

11. M. T. Htay, M. Itoh, Y. Hashimoto, K. Ito, in Proceedings of the 2007 International Symposium on Organic and Inorganic Electronic Materials and Related Nanotechnologies, Nagano, Japan, June 2007, p. 26

12. M. T. Htay, M. Itoh, Y. Hashimoto, K. Ito, Jpn. J. Appl. Phys. 47, 541 (2008) 

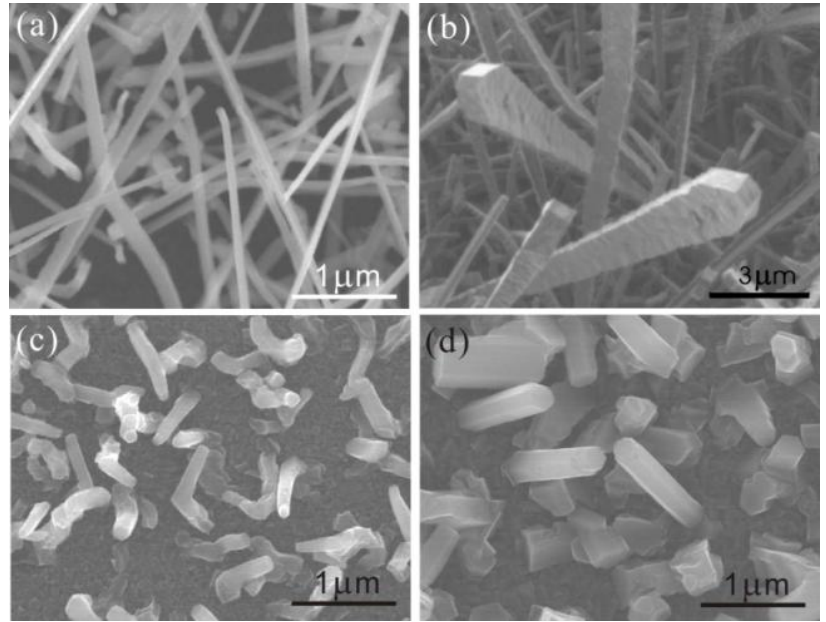

Fig. $1 \mathrm{ZnO}$ nanowires grown for $120 \mathrm{~min}$ at (a)

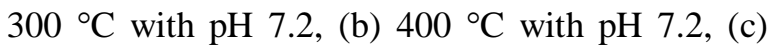
$300{ }^{\circ} \mathrm{C}$ with pH 6.2, and (d) $400{ }^{\circ} \mathrm{C}$ with pH 6.2.
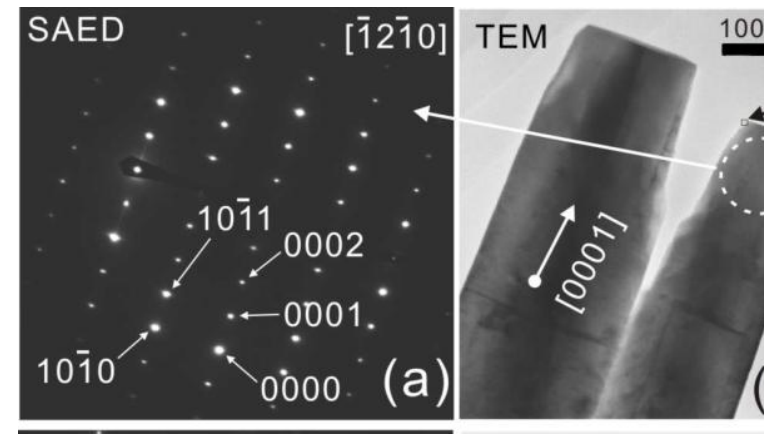

$00 \mathrm{~nm}$ HRTEM
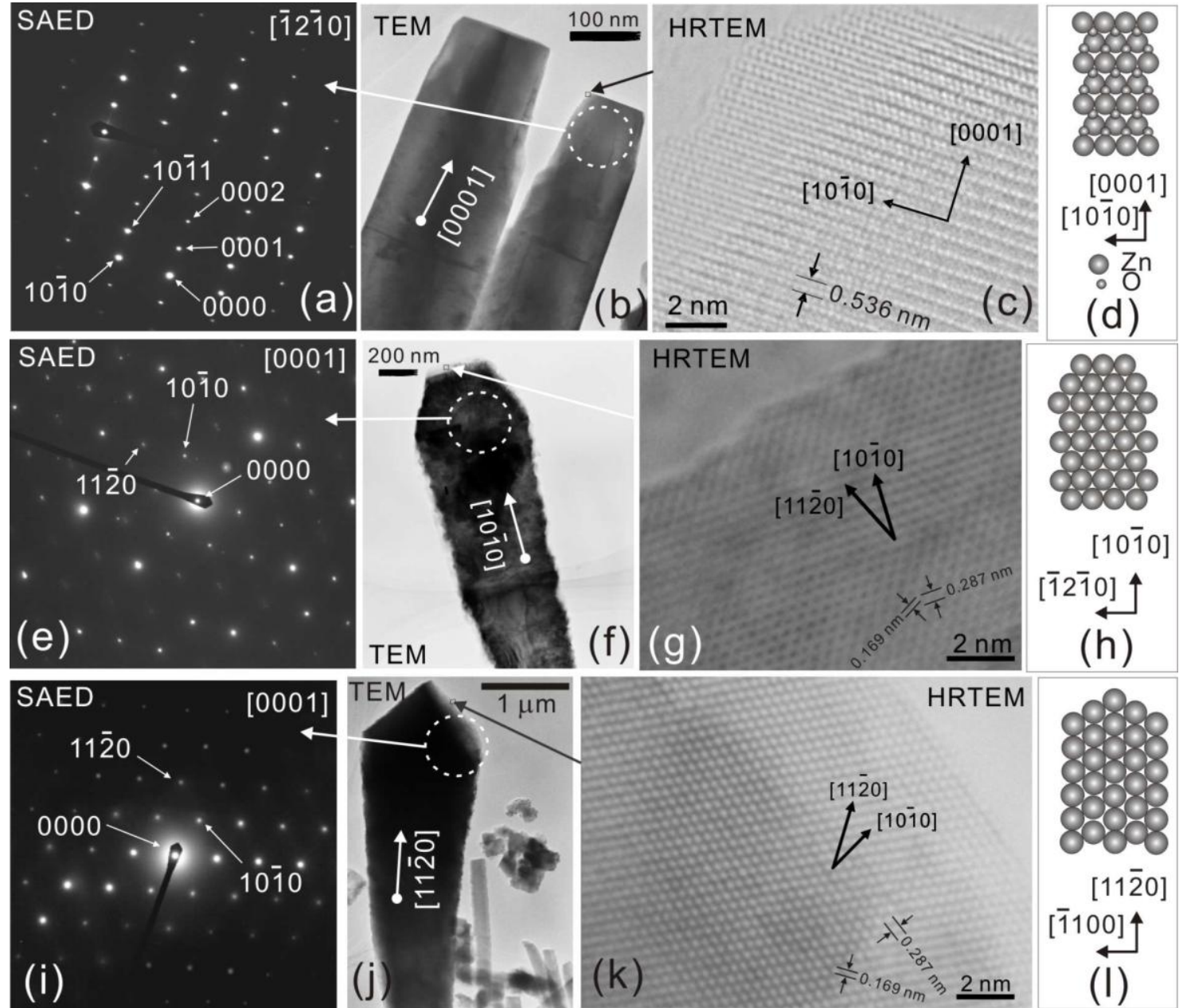

Fig. 2 (a) SAED pattern of encircled area shown in (b). (b) Bright field TEM image of type A wire. (c) HRTEM image of arrowed-region in (b). (d) Atomic topology of type A wire observed from [ $\overline{12} \overline{10}$ ] zone axis. (e) SAED pattern of encircled area shown in (f). (f) Bright field TEM image of type B wire. (g) HRTEM image of arrowed-region in (f). (h) Atomic topology of type B wire observed from [0001] zone axis. (i) SAED pattern of encircled area shown in (j). (j) Bright field TEM of type $\mathrm{C}$ wire. (k) HRTEM image of arrowed-region in (j). (l) Atomic topology of type $\mathrm{C}$ wire observed from [0001] zone axis. 


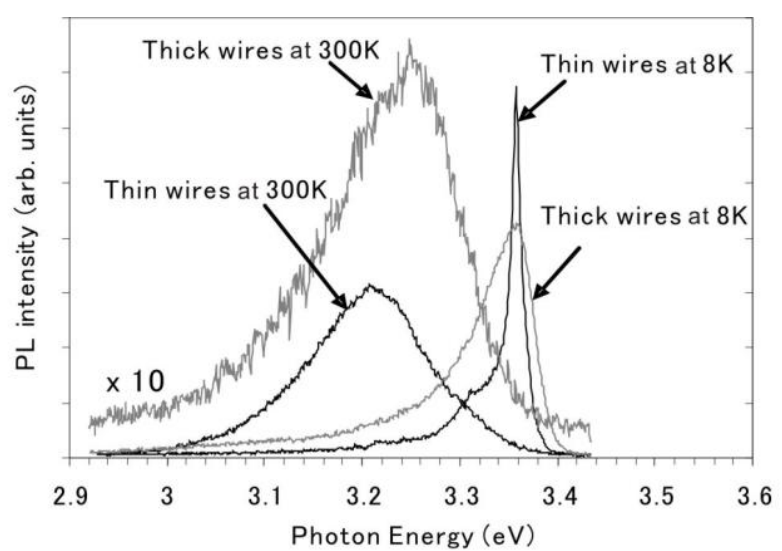

Fig. 3 PL spectra of thin and thick $\mathrm{ZnO}$ wires measured at 8 and $300 \mathrm{~K}$. The intensity of thick wires at $300 \mathrm{~K}$ is multiplied by 10 to enhance the visual.

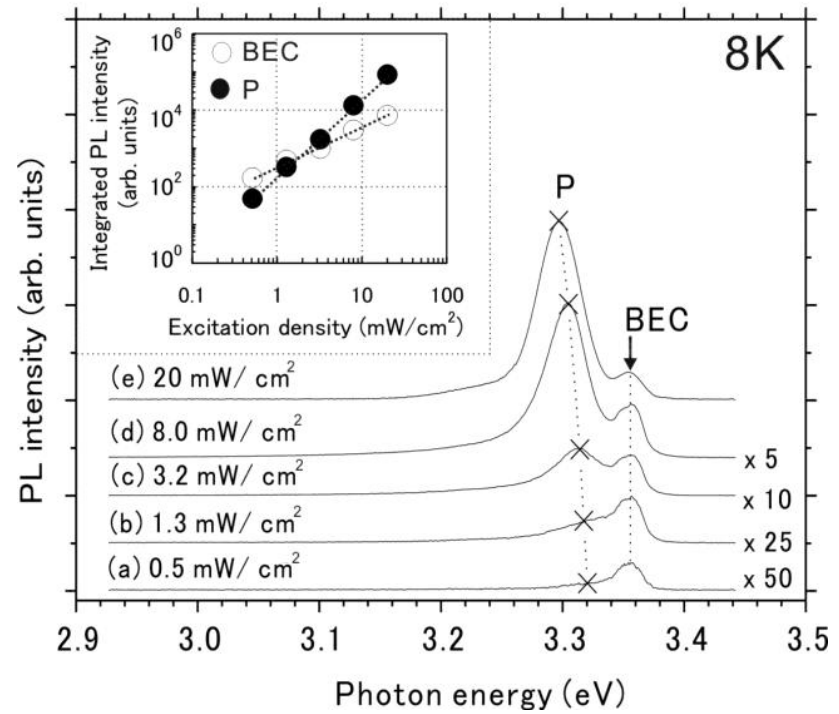

Fig. 4 The excitation-density dependent PL spectra of $\mathrm{ZnO}$ nanowires with an average thickness of 100 $\mathrm{nm}$ measured at $8 \mathrm{~K}$. The intensities of spectra obtained at lower excitation density are multiplied by an arbitrary factor to enhance the visual. The inset shows a log-log plot of the integrated PL intensities of BEC (O) and P-band ( $\bullet$ emissions against the excitation density. 